\title{
Specifically Targeted Electromagnetic Fields Arrest Proliferation of Glioblastoma Multiforme U-87 Cells in Culture
}

\author{
CARMEN J. NARVAEZ ${ }^{1,2}$, SAMANTHA K. MALL ${ }^{2,3}$, AARON FOUNTAIN $^{1,3,4}$, BRIAN A. PARR $^{1,2}$, \\ SRIDAR V. CHITTUR ${ }^{1,2}$, BORIS I. KOKORIN ${ }^{3, \dagger}$, STEPHEN F. BOTSFORD ${ }^{3}$ and JOSEPH F. STARTARI ${ }^{3}$ \\ ${ }^{1}$ Center for Functional Genomics, Rensselaer, NY, U.S.A.; \\ ${ }^{2}$ University at Albany, Rensselaer, NY, U.S.A.; \\ ${ }^{3}$ Anapole Technologies Inc., Burlington, IA, U.S.A.; \\ ${ }^{4}$ Department of Chemistry and Biology, Ryerson University, Toronto, Canada
}

\begin{abstract}
Background/Aim: Glioblastoma multiforme is an aggressive primary tumor that arises in the glial cells of the brain. Standardized first-line treatment has considerable morbidity and less than one-year median survival after intervention. Ultra-low intensity electromagnetic fields have been shown to interact with biological organisms without anticipated deleterious side-effects. The aim of the study was to determine if a novel, non-invasive application of nonionizing radiation has an inhibitory effect on proliferation of glioblastoma multiforme cells. Materials and Methods: U-87 $M G$ cells were continuously exposed for $54 h$ to an electromagnetic field tuned to simultaneously interact with DNA/RNA oligonucleotides (mutated alpha-kinase 2 gene/HsamiR-381-5p respectively) and proteins (HSP70/CHI3L1). Results: Exposed cells demonstrated a significant inhibition of cell growth and concurrent increase in cell death. Conclusion: This technology induces cell death by novel non-cytotoxic mechanisms unlikely to induce side-effects in patients; can be customized for individual tumors and may contribute to the emerging strategy of personalized medicine.
\end{abstract}

Glioblastoma multiforme (GBM) is an aggressive primary tumor that arises in the glial cells of the brain, and accounts for approximately half of all brain tumors. GBM is relatively rare with an incidence of approximately 3 cases per 100,000

This article is freely accessible online.

$\dagger$ Deceased.

Correspondence to: Joseph F. Startari, 1883 Temblethurst Road, South Euclid, Ohio 44121, U.S.A. Tel: +1 8886902091, e-mail: rgfields@anapoletechnologies.com

Key Words: DNA Fragmentation, apoptosis, RGFields ${ }^{\mathrm{TM}}$, resonance, cell cycle, transcriptome. person-years; however, the aggressive nature of the disease and the limited treatment options combine to negatively impact overall survival. Current standardized first-line treatment for GBM consists of surgical resection (debulking), followed by 6 weeks of radiotherapy and concurrent chemotherapy with the alkylating agent, temozolomide (1). Even with this aggressive regimen, which is associated with considerable morbidity, median survival after standard primary intervention is less than a year (2), and less than $5 \%$ of patients survive to 5 years. Since many GBM tumors are resistant to temozolomide due to methylation by O6-methylguanine DNA methyl transferase (MGMT) (3), there is a continuing search for new chemotherapeutic agents that can induce apoptosis in GBM tumors. Possible second line treatments include intralesional implantation of carmustine wafers $(4,5)$ or antiangiogenic therapies with a humanized monoclonal antibody targeting the HGF/SF:cMet axis (6). Pulsed electromagnetic fields in combination with temozolomide have been shown to be proapoptotic (7). A novel method using low intensity, intermediate frequency alternating electric fields (TTFields) to induce cell cycle arrest and cell death in tumors (8) has also recently been approved as a primary treatment for GBM.

Here a novel non-invasive, technology is described, that employs non-ionizing, self-tuned, electromagnetic (EM) radiation that can be added to the therapeutic armamentarium for GBM, alone or in combination with standard therapies. Targeted molecules or cells (reference materials) are selected by identifying critical oncogenic processes in subsets of tumors. These reference materials are then isolated, prepared, stabilized and used to target primary glioblastomas (as modeled in the experiments outlined below). The resonant frequencies of the reference materials are then amplified. These resonances are transmitted to the target cells which alter the behavior of the corresponding reference molecules within the target cells, inducing mitotic arrest and cell death. This system, termed Resonance Generating Fields ${ }^{\mathrm{TM}}$ (RGFIELDS ${ }^{\mathrm{TM}}$ ) technology, can be customized for individual tumors. 
The device used in these experiments emits ultra-low intensity ( -50 to $-90 \mathrm{dBm})$, broadband $(100 \mathrm{MHz}-3.5 \mathrm{GHz})$ EM radiation. Using an array of reference antennae and transceivers coupled to a binary broadband and single frequency waveform generator, the system produces a nonpropagating EM field in the near-field zone of the antennae. The field is self-tuned by means of oscillating waveforms to capture the resonant frequency and harmonics of the selected reference materials. The prescribed reference material resonances are then amplified within the positive feedback loop leading to the disruption of cellular homeostasis, and induction of cell death.

Unlike ionizing radiation, which is widely used in cancer therapy, this technology employs non-ionizing, ultra-low power EM radiation that is not associated with significant side-effects (9). Thus, RGFIELDS ${ }^{\mathrm{TM}}$ offers the promise of an exciting, novel method of cancer therapy with minimal sideeffects that can become part of the emerging personalized medicine approach to combating disease.

\section{Materials and Methods}

Cell culture and growth. U87-MG cells (ATCC ${ }^{\circledR}$ HTB-14 ${ }^{\mathrm{TM}}$ ) were purchased from the American Type Culture Collection (Rockville, MD, USA) and grown in Minimum Essential Medium with Earle's salts and non-essential amino acids (Gibco/Life Technologies, Grand Island, NY, USA) containing 10\% fetal bovine serum (FBS) (Atlanta Biologicals, Norcross, GA, USA) and $1 \mathrm{mM}$ sodium pyruvate (Gibco). U87-MG cells were routinely passaged weekly at a density of 5000 cells $/ \mathrm{cm}^{2}$ with media changes every 3 or 4 days, and maintained in a humidified $5 \% \mathrm{CO}_{2}$ incubator at $37^{\circ} \mathrm{C}$.

Experimental set up. To minimize inadvertent exposure of laboratory personnel to the electromagnetic fields generated during these experiments, the apparatus was placed within a Faraday chamber (Universal Shielding Corporation, Deer Park, NY, USA, Model USC-26, sized $3.2 \mathrm{~m} \times 2.5 \mathrm{~m} \times 2.5 \mathrm{~m}$ ). This shielding provides an attenuation of $100 \mathrm{~dB}$ to electric fields and plane waves from $14 \mathrm{KHz}$ to $10 \mathrm{GHz}$ and $50 \mathrm{~dB}$ to magnetic fields at 14 $\mathrm{KHz}$ rising to $90 \mathrm{~dB}$ at $200 \mathrm{KHz}$ (tested in accordance with IEEESTD-299).

Appropriate $\mathrm{CO}_{2}$ levels were maintained by continuous flow of a $5 \% \mathrm{CO}_{2} / 95 \%$ air mixture, via separate acrylic rotometers (Omega Engineering, Norwalk, CT, USA Model FL 4213).

Custom-designed culture boxes (CCMI, Geneva, NY, USA, $74 \mathrm{~cm}$ $\mathrm{L} \times 48 \mathrm{~cm} \mathrm{~W} \times 38 \mathrm{~cm} \mathrm{H}$ ) consisted entirely of acrylic to minimize interference with the EM fields. Temperature/humidity readings inside each culture box (control and treated) were recorded separately and on a continuous basis (60 sec intervals) using Ethernet data recorders (Omega Engineering, Norwalk, CT, USA, Model iTHX-SD).

Incubator temperatures were maintained separately using individually controlled circulating heaters (Anova Scientific, Stafford, TX, USA, Model C6) and a polycarbonate heating grid (Parkland Scientific, Coral Springs, FL, USA, Model A2788A, $61 \mathrm{~cm} \mathrm{~L} \times 38 \mathrm{~cm}$ $\mathrm{W} \times 3 \mathrm{~cm} \mathrm{H}$ ) located inside of each culture box. Three $150 \mathrm{~mm}$ water filled dishes were positioned on each heating grid as a humidity source (Figure 1).
Prior to inserting any culture dishes into each culture box incubator, temperature and humidity readings were stabilized $\left(36.5^{\circ} \pm 1^{\circ} \mathrm{C}\right.$ and $75 \%$ Humidity $\pm 5 \%$ ). During the stabilization period, background scans were taken with the RGFIELDS ${ }^{\mathrm{TM}}$ equipment in operation ensure proper field strength/tuning and for comparing spectral shifts during actual cell culture exposure against the two background spectra.

Once temperatures/humidity stabilized in both the treated (exposed) and control (unexposed) culture box incubators; and, spectral background scans completed, cell cultures were then inserted into their respective incubator and each maintained for 54 $\mathrm{h}$ continuously. Unexposed controls, as stated above, were maintained separately and via support equipment identical to that used with treated cultures.

Targeted electromagnetic field system description. A schematic of system used to induce an effect by means of targeted electromagnetic radiation at non-ionizing wavelengths is shown in Figure 2 which illustrates the system utilizing a single reference material. Multiple reference materials require parallel circuitry for each broadcast antenna/shielded container added.

Such a system should not be utilized without the protection of an EMF/RFI shielding enclosure when targeting substances produced within the human body. Selection of appropriate targets is critical to successful treatment of a particular disease or malady. In order to achieve the desired effect, the actual targeted material (source or reference material) must be used to capture the appropriate resonant frequencies.

The system includes a selected source material (reference material) or multiple reference materials to target, properly positioned within a shielded container between driven and receiving broadband compatible antennas; a multi-channel signal generator (broadband and single frequency) which supplies modulated signals to the radiating (broadcast) antenna(s); and at least one, broadband capable, feedback antenna which receives radiated signals and supplies said signals back to the shielded container to induce feedback resonance within the reference material(s) in the shielded container(s). These resonant frequency signals are transmitted by cable to the broadcast antenna(s); then amplified by signals from the signal generator; and transmitted subsequently through the broadcast antenna(s) to create a resonance generating EM field. This field induces amplified feedback resonance within the cells, animals, or patients which host each of the selected target or targets.

The transmitted signals supplied by the signal generator (single frequency and broadband) are modulated separately at two or more different frequencies (e.g. $4 \mathrm{~Hz}, 2 \mathrm{kHz}$ ) and at each frequency with a different wave form. This device operates in a non-ionizing frequency range bandwidth of $100 \mathrm{MHZ}$ to $3.5 \mathrm{GHZ}$ at low field emission levels $(0.003 \mathrm{~V} / \mathrm{m}$ or less).

The signal generator supplies a first booster signal at a single frequency $(4 \mathrm{~Hz})$ and a second broadband signal. The broadband signal is modulated by 2 different waveforms, one waveform identical to the first booster signal waveform (a sine wave) at the frequency described previously; and the second waveform (a sawtooth wave) at a different frequency $(2 \mathrm{kHz})$.

The signal generator provides singular and binary output through the broadcast (radiating) antennas to the feedback antenna. This positive feedback is transmitted through the reference material antenna to induce resonances specific to each reference material. These resonances are fed to each of the broadcast antennas and comingled with the transmissions from the signal generator. 


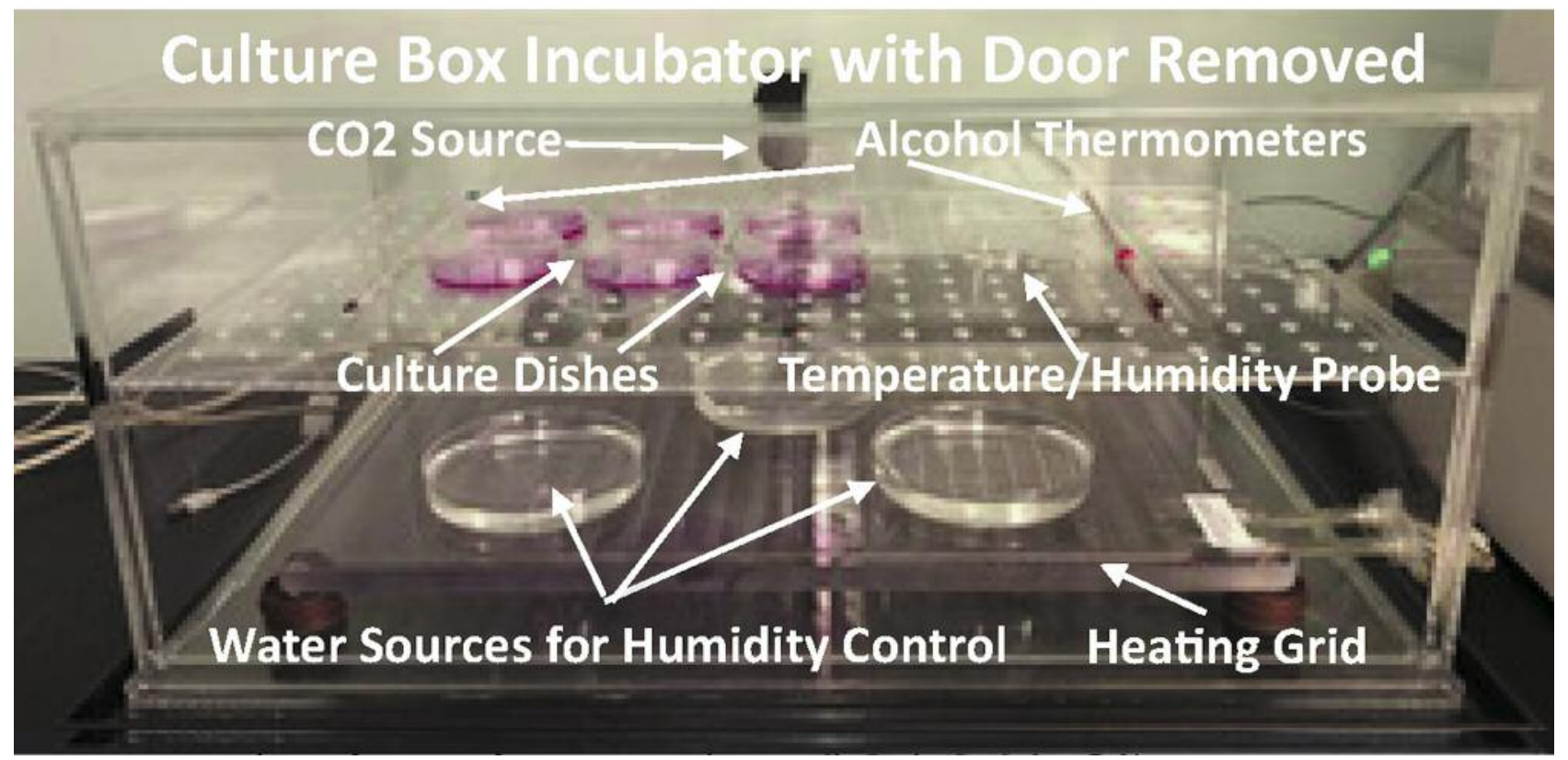

Figure 1. Culture box incubator with door open.

The repetitive feedback action amplifies resonances specific to the source material and quickly causes the external electromagnetic field which forms between the feedback antenna and the radiating antennas (near field zone of the antenna array) to reach steady state (field is now considered to be tuned); which may be confirmed by use of a monitoring antenna and spectrum analyzer. Prolonged exposure of any substance or organism that contains the selected source material(s) induces amplified resonance within the targeted areas.

Modulation of these fields should excite cyclotron and other resonances; wherein prolonged exposure to amplified resonances may produce an effect which is measurable, such as a change in spectra and/or biological activity (Figure 3 ).

Reference material selection, preparation and stabilization. Substances (reference materials) critical to the function or progression of a disease must be identified for targeting. Selection of the appropriate target(s) is the most important requirement to achieve successful results. These reference materials are then isolated and individually placed in separate vials which are nonconductive (such as glass, etc.) by standard laboratory techniques. Only a small amount is required (e.g. less than $1 \mathrm{mg}$ ).

For the proof of principle studies described in this manuscript, four macromolecules were selected as reference materials: hsamiRNA-381 (10); mutated alpha-kinase 2 gene (11); HSP70 (70kDa heat shock protein) and CHI3L1 (chitinase-3-like protein 1) (12).

DNA and RNA oligonucleotides were synthesized and purified by HPLC (Integrated DNA Technologies, Inc; Coralville, IA, USA). The oligonucleotides were solubilized, their volumes reduced and transferred to an appropriate container (vial) for stabilization.

Hsa-miR-381-5p (MIMAT0022862) sequence is 5'AGCGAGGUUGCCCUUUGUAUAU-3'. This microRNA is highly expressed in brain tumors and has recently been identified to play a major role in glioma progression by targeting and inactivating LRRC4 (leucine-rich repeat C4), a tumor suppressor gene that is specifically expressed in brain (9). Targeting this microRNA in U87 MG cells should significantly inhibit cell viability, proliferation and up-regulate expression of the tumor suppressor LRRC4.

The U-87 MG cell line has a homozygous mutated alpha-kinase 2 gene (hg18:uc002lhj.2, uc002lhk.1) with a 15bp sequence 5'AGGACACATCAACTG-3' deleted from the gene (11). The DNA oligonucleotide 5'-AGGGAGACTG^ ${ }^{\wedge}$ TTACCATTGC-3' contains 10 bp of the wild type alpha-kinase 2 coding sequence flanking both sides of the deletion site. The relatively large DNA mutation and its potential contribution to loss-of-function make this gene a suitable candidate as a DNA target.

Recombinant proteins (HSP70 and CHI3L1) were solubilized in PBS buffer. The buffer was exchanged, volume reduced, and transferred to an appropriate container (vial) for stabilization. The human recombinant protein HSP70 $(200 \mu \mathrm{g})$ was purchased from Enzo Life Sciences, Farmingdale, NY, USA. Heat shock proteins are expressed in all cells, and are more highly expressed in cancer cells, due to the protection needed from the stress of their high metabolic requirements (13). In U-87 MG, HSP70 interacts with ATF5, an activating transcription factor, and stabilizes the protein from degradation (14). In other cancers, HSP70 protects antiapoptotic proteins while suppressing pro-apoptotic activity (13). Targeting HSP70 should sensitize U-87 MG to chemical and oxidative stress, radiation therapy, as well as reduce stability of certain survival proteins. The human recombinant his-tagged protein CHI3L1 $(50 \mu \mathrm{g})$ solubilized in PBS was purchased from Invitrogen. Chitinase-3-like protein 1 is a secreted glycoprotein that is overexpressed in glioblastomas and is highly overexpressed in $\mathrm{U}-87 \mathrm{MG}$ cells (15). This protein is pro-angiogenic, contributes to 


\section{Targeted Electromagnetic Field System}

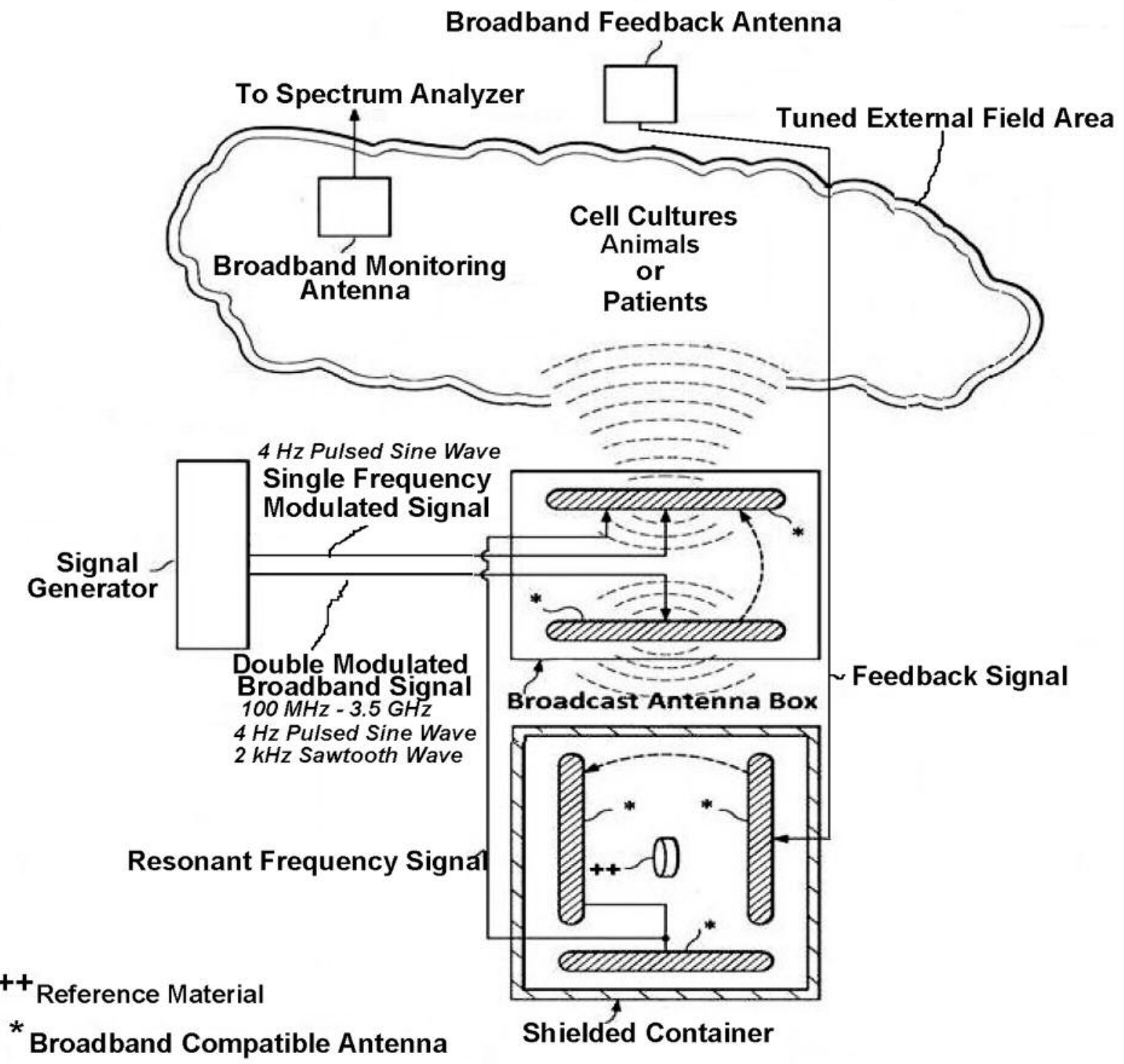

Figure 2. Schematic of Targeted Electromagnetic Field System.

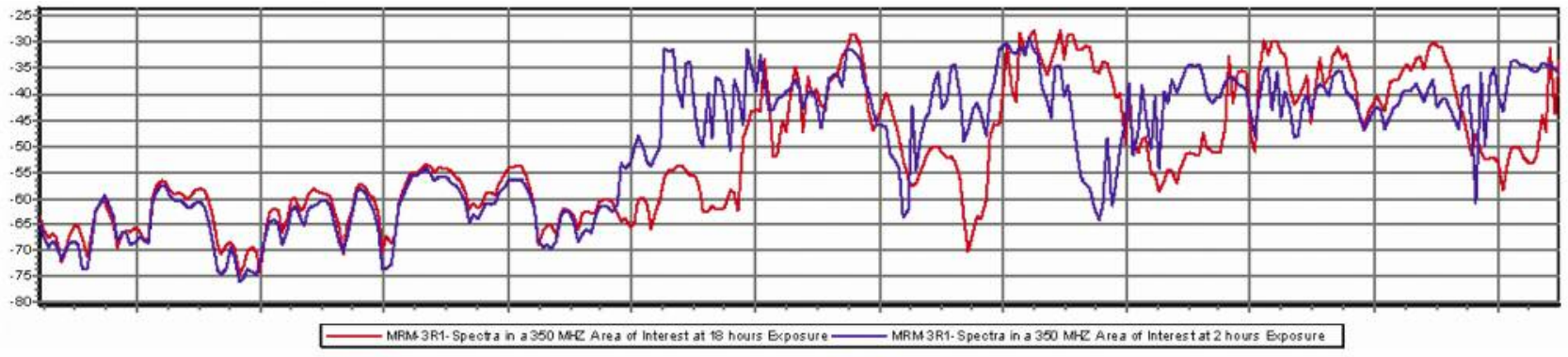

Figure 3. Change in spectra of U-87 MG cells exposed for $2 h$ (blue) vs. those same cultures after being exposed for $18 \mathrm{~h}$ (red). Spectral changes inside the RF/EMI Shield Room (Faraday Chamber) in a $350 \mathrm{MHZ}$ area of interest from U-87 MG cells exposed to RESONANCE GENERATING FIELDS ${ }^{T M}$ tuned for four, U-87 MG macromolecules. 


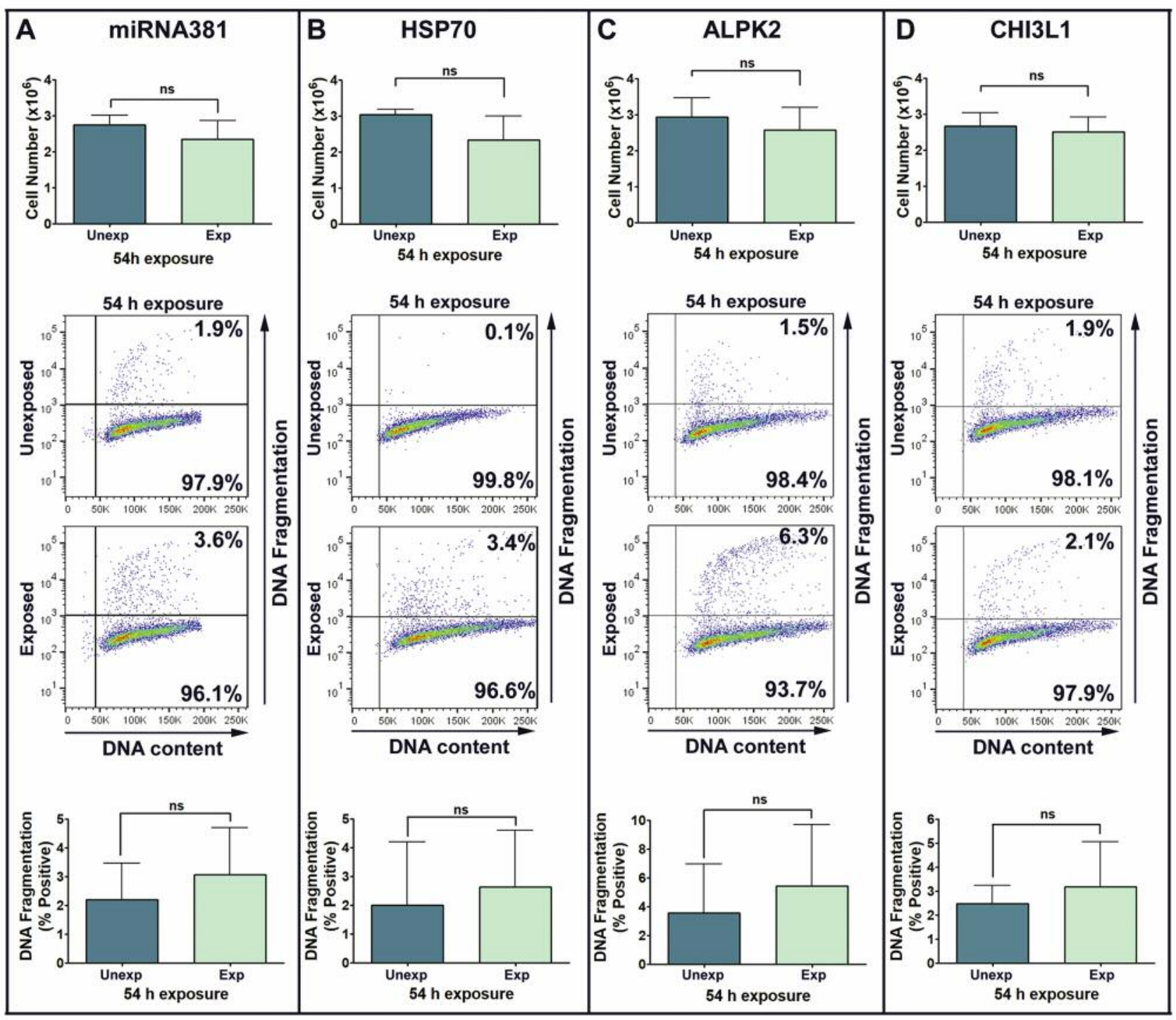

Figure 4. Analysis of U-87 MG cell was proliferation and apoptosis after exposure to RGFIELDS ${ }^{T M}$ at fast modulation using a single reference material. U-87 MG cells were plated as described in Material and Methods and exposed to fast modulation RGFIELDS ${ }^{T M}$ for $54 \mathrm{~h}$ using the following single reference materials individually: Panel A, miRNA381; Panel B, HSP70; Panel C, ALPK2; and Panel D, CHI3L1. At the end of exposure, cells were harvested by trypsinization, and an aliquot of live/unfixed cells were used to determine cell number using the MUSE Cell Analyzer as described in Materials and Methods. The cells were also fixed for DNA fragmentation analysis as described in Materials and Methods. Unexposed control cells were cultured under identical conditions outside the Faraday chamber. Top: Comparison of changes in U-87 MG cell number in control cells and cells after exposure to RGFIELDS ${ }^{T M}$. Data are represented as Mean $\pm S D$ of three independent biological experiments (ns-not significant). Comparison of DNA fragmentation in U-87 MG cells in control cells and cells exposed to RGFIELDS ${ }^{T M}$. Middle: Representative histogram of DNA fragmentation shows percent of cells with intact DNA (lower quadrant) and fragmented DNA (upper quadrant). Bottom: Data, expressed as the percentage of the cell population with fragmented DNA, are shown as Mean $\pm S D$ of three independent biological replicates (ns-not significant).

radio-resistance and tumor progression in glioblastomas (12). Targeting CHI3L1 should decrease proliferation and metastasis in U-87 MG cells.

A mold to contain melted paraffin is required to form a capsule around the reference material source vial. Each vial should then be centered in the melted paraffin in each mold respectively; and held in position with forceps until the paraffin has hardened slightly (enough to prevent further movement). The forceps should then be removed and the paraffin permitted to harden completely.

To ensure proper positioning of the stabilized vial within the shielded container, the capsule is centered between the driven antenna and the two receiving antennas; and the Targeted Electromagnetic Field System is ready for use. We have trademarked this system as Resonance Generating Fields ${ }^{\mathrm{TM}}$ (RGFIELDS ${ }^{\mathrm{TM}}$ ). 
A

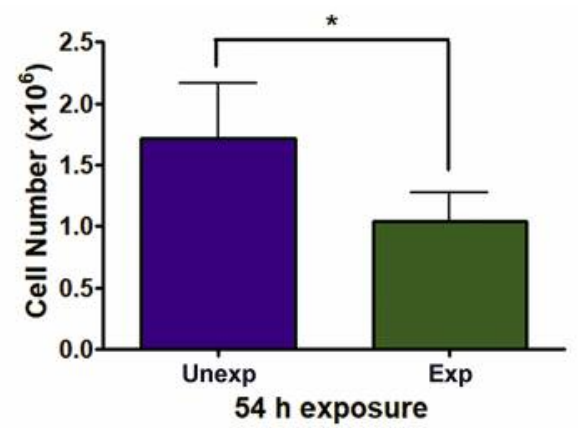

B

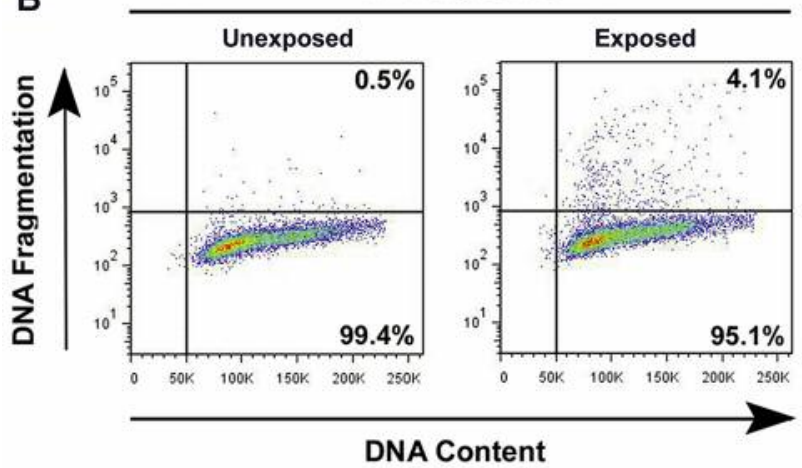

C

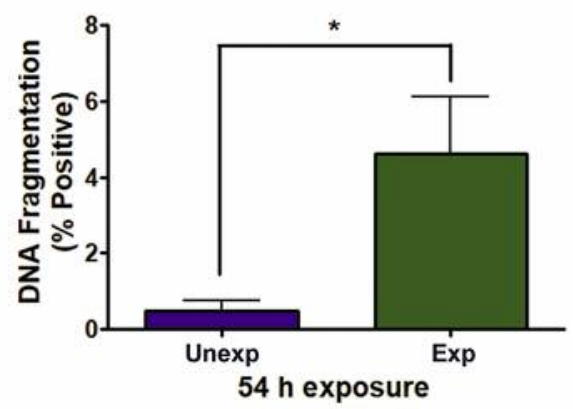

Figure 5. Analysis of $U-87 M G$ cell proliferation and apoptosis after exposure to RGFIELDS ${ }^{T M}$ at fast modulation using all four reference materials simultaneously. U-87 MG cells were plated as described in Material and Methods, and exposed to fast modulation RGFIELDS ${ }^{T M}$ for $54 \mathrm{~h}$. At the end of exposure, cells were harvested by trypsinization, and an aliquot of live/unfixed cells was used to determine cell number using the MUSE Cell Analyzer as described in Materials and Methods. The cells were also fixed for DNA fragmentation analysis as described in Materials and Methods. Control cells were cultured under identical conditions outside the Faraday chamber. Panel A: Comparison of changes in U-87 MG cell number in control cells and cells after exposure to RGFIELDS ${ }^{T M}$. Data are represented as Mean $\pm S D$ of three independent biological experiments $\left({ }^{*} p<0.1\right)$. Comparison of DNA fragmentation in U-87 MG cells in control cells and cells exposed to RGFIELDS ${ }^{T M}$. Panel B: Representative histogram of DNA fragmentation shows percent of cells with intact DNA (lower) and fragmented DNA (upper). Panel C: Data, expressed as the percentage of the cell population with fragmented DNA, are shown as Mean $\pm S D$ of three independent biological replicates $(* p<0.1)$.
RGFIELDS ${ }^{T M}$ calibration, operation and measurement. After the Faraday chamber was installed and tested for compliance by the manufacturer, various spectral scans were run to calibrate the overall system and confirm performance when RGFIELDS ${ }^{\mathrm{TM}}$ were in use.

During this initial system calibration, a series of spectral readings (electromagnetic field intensity) were measured continuously with a spectrum analyzer (Rohde and Schwarz, FSEA Model 20 with a frequency range between $9 \mathrm{kHz}$ and $3.5 \mathrm{GHz}$ ) via a second or higher order, broadband monitoring antenna. These measurements (standard readings using the peak hold function) were recorded by SPAN32 software (internally designed) which facilitates operating the spectrum analyzer from a personal computer or laptop. This software records each scan and enables direct comparison of any scans conveniently throughout, as well as after completion of, an experiment.

The three recorded, calibration measurements were: (i) background radiation was taken outside of the Faraday chamber; (ii) the field intensity inside the Faraday chamber with the equipment on and chamber door sealed; and, (iii) the field intensity inside the Faraday chamber with the equipment on, U-87 MG cell cultures in the treatment culture box and the chamber door sealed. The increase in field intensity in areas of resonance confirmed the system was operating properly and was ready for experimental use.

These measurements also demonstrated that the electric field strength inside the Faraday chamber with the equipment operating was far below the guidelines issued for safe exposure to the general public (16).

Each individual experiment followed standard operating and measurement procedures; which involved two different background scans and then multiple spectral scans throughout the period of exposure to ensure proper operation and response.

Continuous measurement of the broadband spectra of the EM Field, confirmed proper operation of the system; facilitated the observation and analysis of changes in areas showing enhanced resonance response; and documented spectral correlation percentages during each iteration of these studies.

Prior to exposing selected cultures to RGFIELDS ${ }^{\mathrm{TM}}$, the appropriate reference material or materials were installed inside the reference material antenna(s); and subsequently, an initial series of spectral background scans were run.

After observing proper operation during the initial background scans, a $100 \mathrm{~mm}$ Petri dish containing EMEM media was inserted into the culture box and a second series of spectral background scans were conducted.

Once the second background scans were complete; SPAN32 software confirmed adequate tuning ( $85 \%$ scan correlation or greater); and temperature/humidity was within specification; cultures containing U-87 MG cells were then inserted into the treated culture box incubator and exposed to RGFIELDS ${ }^{\mathrm{TM}}$ continuously for $54 \mathrm{~h}$. Similarly, cultures were inserted into the control (unexposed) culture box incubator and maintained continuously for $54 \mathrm{~h}$.

Proper operation of the system was again confirmed by continuously measuring the broadband spectra of the EM Field during the entire $54 \mathrm{~h}$ period of exposure for each iteration of the study.

Anapole's US patent 9610458 issued 4 April 2017 and international application PCT/US2017/036443 was submitted 7 June 2017.

RGFIELDS ${ }^{T M}$ application in vitro. U-87 MG cells were plated at a density of $4 \times 10^{5}$ cells/ 100 -mm dish. $24 \mathrm{~h}$ after seeding, the plates were randomized to the treatment culture box inside the Faraday chamber and exposed to the RGFIELDS ${ }^{\mathrm{TM}}$, or to the control culture box outside the Faraday chamber, to serve as the unexposed controls. 
Cells were exposed to fast modulation $(4 \mathrm{~Hz})$ for $54 \mathrm{~h}$ continuously using each reference material target individually. At the end of exposure, the dishes were processed and cells were harvested by trypsinization, and an aliquot of live/unfixed cells were used to determine cell number using the MUSE Cell Analyzer as described below. The remaining cells were fixed for cell cycle analysis and DNA fragmentation analysis as described below. Control cells were harvested under identical conditions.

Cells were also exposed to fast modulation $(4 \mathrm{~Hz})$ RGFIELDS ${ }^{\mathrm{TM}}$ for $54 \mathrm{~h}$ continuously utilizing all four targets simultaneously. At the end of exposure, cells were harvested exactly as described for individual targets.

MUSE cell analyzer (cell count and cell cycle). U-87 MG cells, harvested by trypsinization, were pooled with medium plus washes and pelleted by centrifugation. The cells were resuspended in complete media diluted 1:10 with PBS (1\%FBS/PBS). For determination of count and viability, an aliquot of the cell suspension was diluted 1:10 with MUSE Count \& Viability reagent (EMD Millipore, Billerica, MA, USA), which differentially stained viable and non-viable cells based on their permeability to two DNA binding dyes and incubated for $5 \mathrm{~min}$ in the dark at room temperature according to manufacturer's protocol.

For cell cycle analysis, an ethanol-fixed cell suspension was obtained by taking an aliquot of cell suspension, pelleting by centrifugation, resuspending in PBS, and fixing in $70 \%$ ethanol at $-20^{\circ} \mathrm{C}$. The fixed cells were washed in $\mathrm{PBS} / 0.2 \% \mathrm{BSA}$, pelleted by centrifugation, resuspended in MUSE Cell Cycle reagent (Millipore), and incubated for $30 \mathrm{~min}$ in the dark at room temperature according to manufacturer's protocol. The MUSE Cell Cycle reagent is a proprietary formulation containing the DNA intercalating dye propidium iodide and RNAse A.

The multiparametric fluorescent detection of individual cells was performed by MUSE Cell Analyzer (Millipore), a microcapillary flow cytometer equipped with a 532-nm laser, a forward scatter and two fluorescence (YLW 576/26, RED 680/30) detectors. Data were captured and analyzed using MUSE 1.4 analysis software. Postacquisition analysis of MUSE Cell Cycle data was performed using FlowJo 7.6.5 (FlowJo, LLC, Ashland, OR, USA). At the end of $54 \mathrm{~h}$ exposure, cell cycle was determined by incubating the cells in MUSE Cell Cycle reagent, following the manufacturer's protocol.

DNA fragmentation assay. For analysis of DNA fragmentation, U87 MG cells were harvested by trypsinization, collected by centrifugation, fixed in $2 \%$ formaldehyde in PBS, and permeabilized in $70 \%$ ethanol at $-20^{\circ} \mathrm{C}$. DNA strand breaks in cells undergoing apoptosis were indirectly labeled with bromodeoxyuridine (Phoenix Flow Systems, San Diego, CA, USA) by terminal transferase (Roche Diagnostics, Indianapolis, IN, USA) and detected by FITCconjugated monoclonal antibody to bromodeoxyuridine using the APO-BrdU kit according to manufacturer's protocol (BD Biosciences, San Jose, CA, USA). Cells were counterstained with $5 \mu \mathrm{g} / \mathrm{ml}$ propidium iodide (PI; Sigma, St. Louis, MO, USA) containing RNase A (Roche) for detection of total DNA, and twocolor analysis of DNA strand breaks and cell cycle was achieved by flow cytometry.

Flow cytometric analyses were performed on a BD LSR II Flow Cytometer (BD Biosciences) equipped with three excitation lasers (405-nm, 488-nm and 633-nm). For DNA fragmentation analysis, FITC and PI signals were collected by $488-\mathrm{nm}$ octagon detection array with no color compensation. Ten thousand events gated on single cell populations were collected and post-acquisition analysis was performed using FlowJo 7.6.5.

Transcriptome analysis. For transcriptome analysis, total RNA was isolated from three independent biological replicates of U-87 MG cells exposed to RGFIELDS ${ }^{\mathrm{TM}}$ utilizing all four targets simultaneously for $54 \mathrm{~h}$, and from unexposed control cells cultured under identical conditions using RNeasy Mini kits (Qiagen, Valencia, CA, USA). RNA quality was assessed using both a nanodrop and a Bioanalyzer (Agilent, Santa Clara, CA, USA); and, RNA samples with RIN $>9$ were used for transcriptome analysis. Genomic profiling was conducted with Affymetrix Human Gene 2.0 ST arrays comprising 48,226 genes and the CEL files were converted to CHP files using RMA in Expression Console 3.0; and, used for statistical analysis (ANOVA $p<0.05$ ) in Transcriptome Analysis Console 3.03 (Affymetrix, Santa Clara, CA, USA). Two separate tables were created by further filtering, which identified genes that were differentially expressed, after $54 \mathrm{~h}$ exposure to RGFIELDS ${ }^{\mathrm{TM}}$ at a 1.5fold or higher level.

These differentially expressed gene data were subsequently mapped to cellular pathways using Ingenuity Pathway Analysis (IPA) (Qiagen, Redwood City, CA, USA) to delineate the probable mechanism of action.

All data are MIAME compliant and the raw data are deposited in Gene Expression Omnibus (GEO) GSE108870.

Statistical analysis. Data are expressed as Mean \pm SD of three independent biological experiments and statistical evaluation was by unpaired $t$-test using GraphPad Prism 4 software (La Jolla, CA, USA) or Excel 2010 Analysis ToolPak (Bellevue, WA, USA). $p$ Values $<0.1$ were considered to be significant.

\section{Results}

Four experiments exposed U-87 MG cells to RGFIELDS ${ }^{\text {TM }}$ for $54 \mathrm{~h}$ continuously, each using a single different reference material as a target (multiple iterations). A fifth experiment series exposed U-87 MG cells to RGFIELDS ${ }^{\mathrm{TM}}$ for $54 \mathrm{~h}$ continuously using all four reference materials as targets simultaneously (multiple iterations). Each of the individually targeted reference materials showed a decrease in U-87 MG cell number in the treated cultures compared to untreated control cultures; however, the effects of RGFIELDS ${ }^{\mathrm{TM}}$ on cell number did not reach significance with any single reference material. The effects of RGFIELDS ${ }^{\mathrm{TM}}$ on DNA fragmentation or Cell Cycle (Figure 4; Table I) were similarly not significant.

To test possible synergistic effects of disrupting multiple target molecules simultaneously, experiments were conducted using all four reference materials simultaneously for $54 \mathrm{~h}$ (Figure 5 and Table II). After $54 \mathrm{~h}$ exposure to RGFIELDS $^{\mathrm{TM}}$ targeting all four reference materials collectively, there was a significant decrease (40\%) in U-87 MG cell number $\left(1.0 \times 10^{6}\right.$ cells/dish $)$, relative to unexposed control cells $\left(1.7 \times 10^{6}\right.$ cells/dish) (Figure 5 Panel A). These data suggest that RGFIELDS ${ }^{\mathrm{TM}}$, tuned to all four reference 
Table I. Cell-cycle analysis of U-87 MG cells after exposure to RGFIELDS ${ }^{T M}$ at fast modulation using the following single reference materials: miRNA381, HSP70, ALPK2, and CHI3L1, independently. $U$ $87 M G$ cells were plated and treated as described in Figure 4. At the end of 54 h exposure, cell cycle was determined by incubating the cells in MUSE Cell Cycle reagent, following the manufacturer's protocol. Data represent the Mean $\pm S D$ of three independent biological replicates.

\begin{tabular}{lcccc}
\hline Single reference material & \multicolumn{4}{c}{54 h exposure } \\
\cline { 2 - 5 } & Sub-G & $\mathrm{G}_{1}$ & $\mathrm{~S}$ & $\mathrm{G}_{2} / \mathrm{M}$ \\
\hline miRNA381 & & & & \\
$\quad$ Unexposed & $0.0 \pm 0.0$ & $50.0 \pm 0.8$ & $21.5 \pm 0.2$ & $29.1 \pm 1.4$ \\
$\quad$ Exposed & $0.0 \pm 0.0$ & $48.0 \pm 3.4$ & $23.4 \pm 3.4$ & $29.8 \pm 6.8$ \\
HSP70 & & & & \\
$\quad$ Unexposed & $0.0 \pm 0.7$ & $50.9 \pm 4.5$ & $22.1 \pm 1.6$ & $26.7 \pm 3.6$ \\
$\quad$ Exposed & $0.0 \pm 0.0$ & $48.6 \pm 4.7$ & $22.2 \pm 1.9$ & $28.9 \pm 6.4$ \\
ALPK2 & & & & \\
$\quad$ Unexposed & $0.8 \pm 0.7$ & $51.5 \pm 2.5$ & $22.2 \pm 2.3$ & $24.6 \pm 1.5$ \\
$\quad$ Exposed & $0.0 \pm 0.0$ & $52.6 \pm 1.7$ & $22.3 \pm 1.3$ & $25.2 \pm 2.0$ \\
CHI3L1 & & & & \\
$\quad$ Unexposed & $0.0 \pm 0.0$ & $52.4 \pm 1.5$ & $22.0 \pm 1.8$ & $25.5 \pm 2.2$ \\
Exposed & $0.2 \pm 0.3$ & $52.2 \pm 2.0$ & $21.0 \pm 0.6$ & $27.6 \pm 3.5$ \\
\hline
\end{tabular}

materials outlined above, arrested cell growth in U-87 MG cells. This decrease in cell number was accompanied by a decrease in the proportion of the cells in the $\mathrm{G}_{1}$ phase of the cell cycle and a concomitant increase in the proportion of cells in $\mathrm{G}_{2} / \mathrm{M}$. This change in cell cycle kinetics was accompanied by the induction of cell death, as measured by DNA fragmentation (Figure 5, Panels B and C), which shows that RGFIELDS ${ }^{\mathrm{TM}}$ induce a large increase in TUNEL positive cells after $54 \mathrm{~h}$ of exposure.

To elucidate the cellular processes involved in the response of U-87 MG to exposure to RGFIELDS ${ }^{\mathrm{TM}}$ generated by all four reference materials simultaneously, the transcriptome of the U-87 MG cells exposed to the RGFIELDS $^{\mathrm{TM}}$ for $54 \mathrm{~h}$ was compared to unexposed cells. Rigorous analysis of the array data determined that 135 mRNA and non-coding RNA sequences were downregulated 1.5-fold or more in response to the RGFIELDS ${ }^{\mathrm{TM}}$, while 178 sequences were up-regulated. More stringent filtering of the above data set (ANOVA $p<0.02$ ) identified only 24 sequences that were up-regulated and 9 sequences that were down-regulated more than 2-fold (Table III).

To assess which cellular pathways are impacted, the gene list of the entities modulated 2.0 or more was further analyzed using IPA. As shown in Figures 6 and 7, the major pathways that were impacted by RGFIELDS ${ }^{\mathrm{TM}}$ appear to regulate cell cycle checkpoint control and the cellular signaling downstream of DNA damage mediated by ATM, the cellular sensor pathway of double strand breaks.
Table II. Cell Cycle analysis of U-87 MG cells after exposure to RGFIELDS ${ }^{T M}$ at fast modulation using all four reference materials simultaneously. U-87MG cells were plated and treated as described in Figure 4. At the end of 54 h exposure, cell cycle was determined by incubating the cells in MUSE Cell Cycle reagent, following the manufacturer's protocol. Data represent the Mean $\pm S D$ of three independent biological replicates $(* p<0.1)$.

\begin{tabular}{lcccc}
\hline & \multicolumn{5}{c}{54 h exposure } \\
\cline { 2 - 5 } & Sub-G & $\mathrm{G}_{1}$ & $\mathrm{~S}$ & $\mathrm{G}_{2} / \mathrm{M}$ \\
\hline Unexposed & $0.2 \pm 0.3$ & $52.9 \pm 3.5$ & $16.3 \pm 4.1$ & $30.7 \pm 7.3$ \\
Exposed & $0.6 \pm 0.3^{*}$ & $49.4 \pm 3.9$ & $16.9 \pm 2.4$ & $32.8 \pm 5.1$ \\
\hline
\end{tabular}

\section{Discussion}

The data presented in this manuscript demonstrate that RGFIELDS ${ }^{\mathrm{TM}}$ tuned to target individual reference materials show a trend of decreasing U-87 MG cell number and do not significantly induce cell-cycle arrest or apoptosis. However, when U-87 MG cells are exposed to RGFIELDS ${ }^{\mathrm{TM}}$ generated simultaneously by all four targets, there is a significant, $40 \%$ reduction in cell number compared to unexposed control cells. This reduction in cell number is concordant with alterations in cell-cycle kinetics and highly significant increases in DNA fragmentation. Exposure of U-87 MG cells to RGFIELDS ${ }^{\mathrm{TM}}$ utilizing all four targets simultaneously significantly increases the sub G0 population (indicative of the formation of apoptotic bodies) and DNA fragmentation suggesting that these electromagnetic fields induce DNA fragmentation and cell death.

While the transcriptome analyses have not been validated by using qPCR or Western analysis, they do provide corroborative evidence that identifies a number of intracellular signaling pathways that may be responsible for the biological endpoints we have characterized in this study. Further studies will clearly be required to confirm the importance of these pathways in the response to RGFIELDS $^{\mathrm{TM}}$. Importantly IPA analysis suggests that RGFIELDS $^{\mathrm{TM}}$ technology induces the same cell cycle and apoptotic pathways as ionizing radiation, UV radiation and some chemotherapeutics, without inducing the significant side effects of these modalities.

Unlike conventional electromagnetic treatments, the use of reference materials captures the exact resonant frequency and harmonics of the target without having to calculate the appropriate resonant frequency or determine an effective resonant frequency for a particular outcome by trial and error. In addition, unlike TTFields, there are unlikely to be unwanted dermatologic side effects (17). 
Table III. Transcriptome Data - Exposure to RGFIELDS ${ }^{T M}$ using all four reference materials simultaneously. Array Data (2-fold changes at ANOVA $p<0.02)$ comparing the transcriptome of the U-87MG cells exposed to the RGFIELDS ${ }^{T M}$ for 54 h to unexposed cells.

\begin{tabular}{|c|c|c|c|c|}
\hline \multirow[t]{2}{*}{ Treated vs. Control } & \multicolumn{2}{|c|}{ Sorted by Fold Change } & \multicolumn{2}{|c|}{ Sorted by Gene } \\
\hline & $\begin{array}{l}\text { Fold Change } \\
\text { (linear) } \\
\text { (Treated } v s . \\
\text { Control) }\end{array}$ & Gene Symbol & Gene Symbol & $\begin{array}{l}\text { Fold Change } \\
\quad \text { (linear) } \\
\text { (Treated } v s . \\
\text { Control) }\end{array}$ \\
\hline \multicolumn{5}{|l|}{ Analysis Type: Gene Level } \\
\hline Differential Expression Analysis & 4.23 & OTTHUMG00000035603 & ACTA2 & 2.02 \\
\hline Array Type: HuGene-2_0-st & 3.4 & PCDH18 & AKAP3 & 2.22 \\
\hline Genome Version: hg19 & 2.83 & PRSS35 & CTSO & -2.08 \\
\hline Annotation File: HuGene-2_ & 2.76 & SLITRK6 & FLJ22447 & 2.28 \\
\hline 0-st-v1.na34.hg 19.transcript.csv & 2.48 & MYH15 & $G B P 2$ & 2.18 \\
\hline Summary: & 2.47 & $M R G P R F$ & $H C F C 1 R 1$ & 2.14 \\
\hline 1.) Total number of genes: 48226 & 2.38 & $T P P 1$ & $H I S T 1 H 2 B D$ & 2.01 \\
\hline 2.) 33 genes are differentially expressed & 2.28 & FLJ 22447 & $I L 36 R N$ & 2.16 \\
\hline 3.) Treated vs. Control: & 2.25 & $T L R 3$ & KRTAP1-5 & -2.21 \\
\hline a. 24 genes are up-regulated & 2.22 & $A K A P 3$ & KRTAP2-3 & -2.35 \\
\hline \multirow[t]{2}{*}{ b. 9 genes are down-regulated } & 2.18 & $G B P 2$ & LINC00641 & -2.09 \\
\hline & 2.16 & $I L 36 R N$ & LOC100130887 & 2.07 \\
\hline Algorithm Options: & 2.16 & P4HA1 & LOC645638 & 2.13 \\
\hline \multirow[t]{2}{*}{ 1.) One-Way Between-Subject ANOVA (unpaired) } & 2.14 & $H C F C 1 R 1$ & LOC654433 & 2.04 \\
\hline & 2.13 & LOC645638 & MANBA & 2.11 \\
\hline Default Filter Criteria: & 2.11 & $M A N B A$ & $M R G P R F$ & 2.47 \\
\hline 1.) Fold Change (linear) $<-2.0$ or Fold Change (linear) $>2.0$ & 2.1 & OTTHUMG00000019252 & MYH15 & 2.48 \\
\hline \multirow[t]{2}{*}{ 2.) ANOVA $p$-value (Condition pair) $<0.02$} & 2.07 & LOC100130887 & NUCKS1 & -2.08 \\
\hline & 2.07 & ZNF404 & OTTHUMG00000019252 & 2.1 \\
\hline Conditions: & 2.05 & UBA7 & OTTHUMG00000035603 & 4.23 \\
\hline Treated (3) & 2.04 & LOC654433 & OTTHUMG00000165727 & 2.02 \\
\hline 1.) TRTB_11112016_(HuGene-2_0-st).rma-gene-full.chp & 2.02 & ACTA2 & P4HAl & 2.16 \\
\hline 2.) TRTC_11112016_(HuGene-2_0-st).rma-gene-full.chp & 2.02 & OTTHUMG00000165727 & PCBP3 & -2 \\
\hline \multirow{2}{*}{ 3.) TRTD_11112016_(HuGene-2_0-st).rma-gene-full.chp } & 2.01 & $H I S T 1 H 2 B D$ & PCDH18 & 3.4 \\
\hline & -2 & РCBP3 & PRSS35 & 2.83 \\
\hline Control (3) & -2 & SNORD114-20 & RBM3 & -2.82 \\
\hline 1.) CTRLB_11112016_(HuGene-2_0-st).rma-gene-full.chp & -2.08 & CTSO & SLITRK6 & 2.76 \\
\hline 2.) CTRLC_11112016_(HuGene-2_0-st).rma-gene-full.chp & -2.08 & NUCKS1 & SNORD114-20 & -2 \\
\hline \multirow[t]{5}{*}{ 3.) CTRLD_11112016_(HuGene-2_0-st).rma-gene-full.chp } & -2.09 & LINC00641 & $T L R 3$ & 2.25 \\
\hline & -2.12 & $U H R F 1 B P 1$ & $T P P 1$ & 2.38 \\
\hline & -2.21 & KRTAP1-5 & $U B A 7$ & 2.05 \\
\hline & -2.35 & KRTAP2-3 & $U H R F 1 B P 1$ & -2.12 \\
\hline & -2.82 & RBM3 & ZNF404 & 2.07 \\
\hline
\end{tabular}

As proof-of-principle we have tested four different macromolecular targets both individually and collectively; while the different targets exhibit a range of effectiveness, it appears that collective use of the targets is additive or synergistic. In the future, optimization of the targets based on genome wide comparisons of the common genetic lesions among glioblastoma tumors may identify one or more combinations of reference materials for treatment of specific subsets of tumors. For example, tumors with isocitrate dehydrogenase $(I D H)$ mutations may be targeted more effectively with reference materials based on the known IDH mutations (18), while tumors known to harbor other mutations may not be affected.
Further studies will be needed to determine whether patients with GBM will require longer term, intermittent or continual exposure to RGFIELDS ${ }^{\mathrm{TM}}$ to stop growth and progression of GBM. There may be an enrichment of glioblastoma stem-like cells among the surviving population (19). These stem-like cells could also be effectively targeted concurrently using reference antennae with OLIG2 and SALL2 as targets to self-tune the RGFIELDS ${ }^{\mathrm{TM}}$ to reduce or prevent the initiation of cell growth (19). In the near future, given the advent of inexpensive whole genome sequencing analysis, it will be possible to obtain individualized deep sequencing data derived from the tumor, essentially using the 


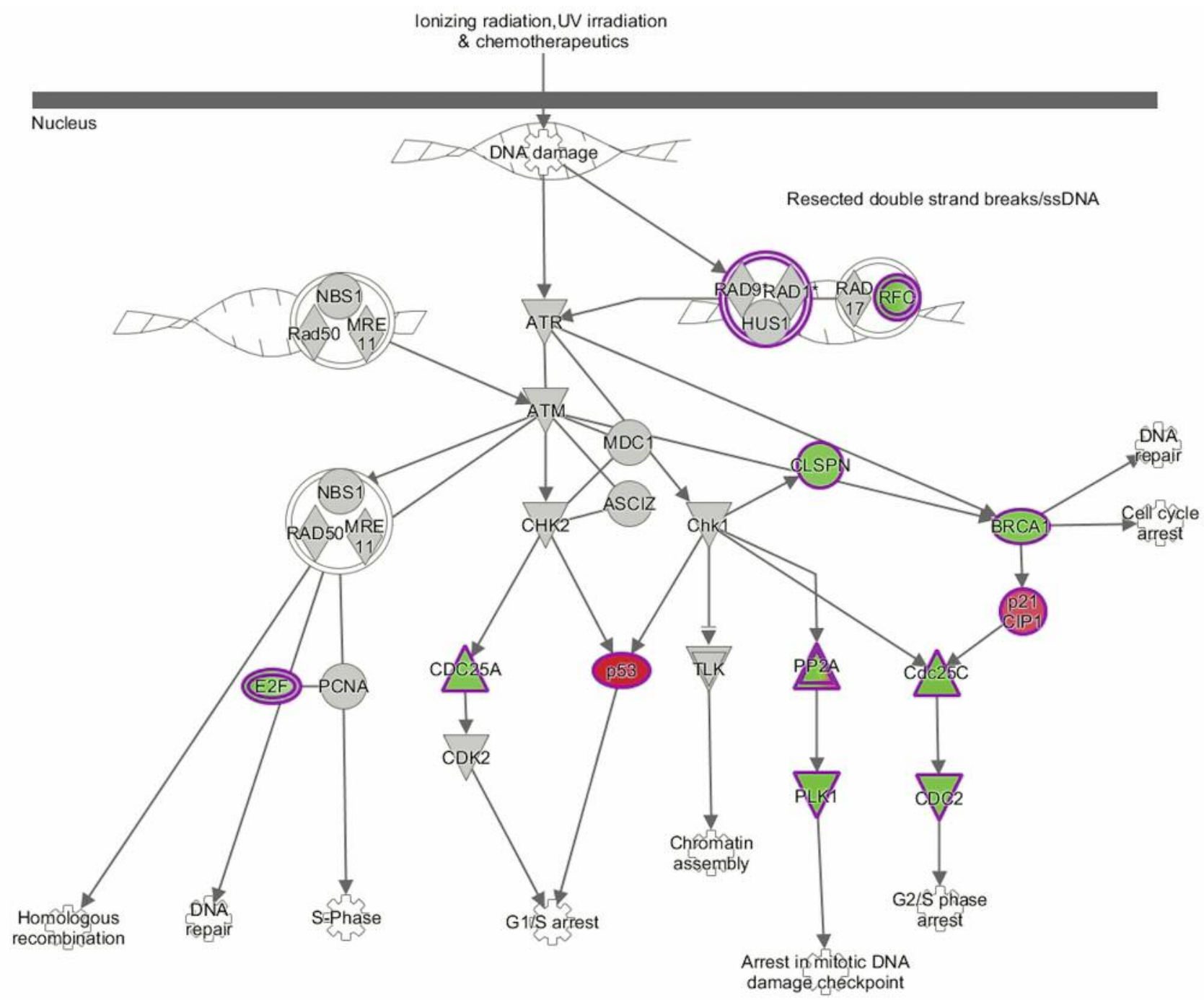

Figure 6. Ingenuity Pathway Analysis displaying regulatory relationships among up-regulated (red) and down-regulated (green) transcripts involved in cell cycle checkpoint control in U-87 MG cells after exposure to RGFIELDS ${ }^{T M}$ using all four reference materials simultaneously. This pathway analysis was generated using IPA under license from Qiagen. The symbols shown are generated by Ingenuity and are standard for the methodology. Uncolored symbols represent transcripts relevant to the pathway but did not pass the analysis cutoffs.

elegant approach described by Koh et al. (20). This would permit the selection of patient specific targets for the reference antennae, which could be used for second line therapy in combination with other modalities. Xenograft studies, using the models similar to that developed by Hashizume et al. (21), are necessary to determine whether control of GBM tumor growth can be achieved with intermittent exposure to RGFIELDS ${ }^{\mathrm{TM}}$.

This technology induces a significant inhibitory effect on U-87 MG cells, likely through a highly significant increase in DNA fragmentation; and, is unlikely to induce toxic or other negative side effects. The targets used to treat cells can, without modification, be immediately used to treat animals and patients; thereby greatly reducing the timeframe from initial in vitro testing through animal studies to patient feasibility studies. Thus, the possibility of repetitive therapy sessions using RGFIELDS ${ }^{\mathrm{TM}}$ has profound implications that may create valuable medical options and a paradigm shift in cancer therapy.

\section{Acknowledgements}

The Authors dedicate this manuscript to Boris I. Kokorin, our colleague and friend, who passed away recently, for his contribution as one of the original inventors of the technology, his desire to make the world a better place for humanity and for his participation in writing this manuscript. The Authors would like to thank Dr. Martin 


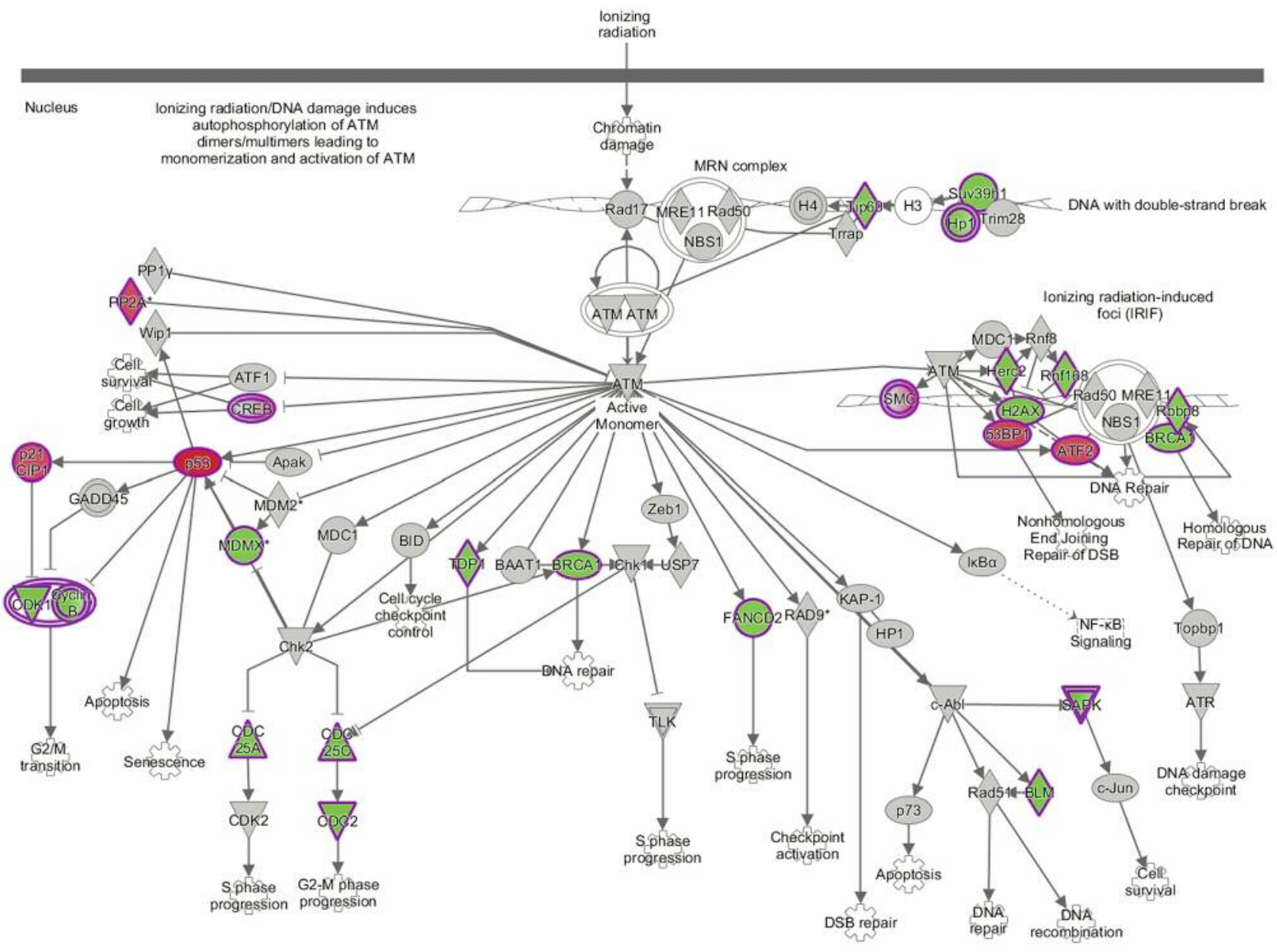

Figure 7. Ingenuity Pathway Analysis displaying regulatory relationships among up-regulated (red) and down-regulated (green) transcripts involved in DNA double strand break/ATM signaling pathways in U-87 MG cells after exposure to RGFIELDS ${ }^{T M}$ using all four reference materials simultaneously. This pathway analysis was generated using IPA under license from Qiagen. The symbols shown are generated by Ingenuity and are standard for the methodology. Uncolored symbols represent transcripts relevant to the pathway but did not pass the analysis cutoffs.

Tenniswood, University at Albany Cancer Research Center, for his excellent advice and his editorial comments. The Authors also thank Dr. Stephen A. Fausel, emeritus, for his vision and support as a founder of, and original benefactor to, Anapole Technologies, Inc.

\section{References}

1 Gallego O: Nonsurgical treatment of recurrent glioblastoma. Curr Oncol 22(4): E273-E281, 2015.

2 Deborah S, Lynch CF, Sibenaller ZA and Ryken TC: Trends in brain cancer incidence and survival in the United States: Surveillance, Epidemiology, and End Results Program, 1973 to 2001. Neurosurg Focus 20(4): E1, 2006.

3 Hegi ME, Hamou M, Gorlia T, Diserens A, Tribolet N, Kros J, Weller M, Hainfellner J, Mason W, Mariani L, Hau P, Bromberg J, Mirimanoff R, Stupp R, Janzer R and Cairncross J: MGMT gene silencing and benefit from temozolomide in glioblastoma. N Engl J Med 352(10): 997-1003, 2005.
4 Duntze J, Litré C, Eap C, Théret E, Debreuve A, Jovenin N, Lechapt-Zalcman E, Metellus P, Colin P, Guillamo J, Emery E, Menei P, Rousseaux P and Peruzzi P: Implanted carmustine wafers followed by concomitant radiochemotherapy to treat newly diagnosed malignant gliomas: prospective, observational, multicenter study on 92 cases. Ann Surg Oncol 20(6): 2065-2072, 2013.

5 Wait SD, Prabhu RS, Burri SH, Atkins TG and Asher AL: Polymeric drug delivery for the treatment of glioblastoma. Neurol Oncol 17(Suppl 2): ii9-ii23, 2015.

6 Jun HT, Sun J, Rex K, Radinsky R, Kendall R, Coxon A and Burgess TL: AMG 102, a fully human anti-hepatocyte growth factor/scatter factor neutralizing antibody, enhances the efficacy of temozolomide or docetaxel in U-87 MG cells and xenografts. Clin Cancer Res 13(22): 6735-6742, 2007.

7 Pasi F, Fassina L, Mognaschi ME, Lupo G, Corbella F, Nano R and Capelli E: Pulsed electromagnetic field with temozolomide can elicit an epigenetic pro-apoptotic effect on glioblastoma T98G Cells. Anticancer Res 36(11): 5821-5826, 2016. 
8 Kirson E, Wasserman Y, Gurvich Z, Schneiderman R, Schatzberger R, Palti Y, Dekel E and Itzhaki A: Disruption of cancer cell replication by alternating electric fields. Cancer Res 64(9): 3288-3295, 2004

9 Gathiram P, Kistnasamy B and Lalloo U: Effects of a unique electromagnetic field system on the fertility of rats. Arch Environ Occup Health 64(2): 93-100, 2009.

10 Tang H, Liu X, Wang Z, She X, Zeng X, Deng M, Liao Q, Guo X, Wang R, Li X, Zeng F, Wu M and Li G: Interaction of hsamiR-381 and glioma suppressor LRRC4 is involved in glioma growth. Brain Res 1390: 21-32, 2011.

11 Clark MJ, Homer N, O'Connor BD, Zugen C, Eskin A, Lee H, Merriman B and Nelson SF: U87MG decoded: The genomic sequence of a cytogenetically aberrant human cancer cell line. PLOS Genetics 6(1): 1-16, 2010.

12 Francescone R, Scully S, Faibish M, Yan W, Shao R, Bentley B, Taylor S, Oh D and Moral L: Role of YKL-40 in the angiogenesis, radioresistance, and progression of glioblastoma. J Biol Chem 286(17): 15332-15343, 2011.

13 Goloudina AR, Demidov ON and Garrido C: Inhibition of HSP70: A challenging anti-cancer strategy. Cancer Lett 325(2): 117-124, 2012.

14 Li G, Xu Y, Guan D, Liu Z and Liu D: HSP70 protein promotes survival of C6 and U87 glioma cells by inhibition of ATF5 degradation. J Biol Chem 286(23): 20251-20259, 2011.

15 Formolo CA, Williams R, Gordish-Dressman H, MacDonald TJ, Lee $\mathrm{NH}$ and Hathout Y: Secretome Signature of Invasive Glioblastoma Multiforme. J Proteome Res 10(7): 3149-3159, 2011.

16 International Commission on Non-Ionizing Radiation Protection (ICNIRP): Guidelines for limiting exposure to time-varying electric, magnetic, and electromagnetic fields (Up To $300 \mathrm{GHZ}$ ). Health Phys 74(4): 494-522, 1998.
17 Lacouture ME, Davis ME, Elzinga G, Butowski N, Tran D, Villano JL, DiMeglio, L, Davies A and Wong E: Characterization and management of dermatologic adverse events with the novoTTF-100A system, a novel anti-mitotic electric field device for the treatment of recurrent glioblastoma. Semin Oncol 41(Suppl 4): S1-S14, 2014.

18 Zeng A, Hu Q, Cui X, Li R, Yan W, You Y, Liu Y and Wang Z: IDH1/2 mutation status combined with Ki-67 labeling index defines distinct prognostic groups in glioma. Oncotarget 6(30): 30232-302328, 2015.

19 Suvà ML, Rheinbay E, Gillespie SM, Patel AP, Regev A, Bernstein BE, Riggi N, Rivera MN, Rossetti N, Louis DN, Beik S, Kadri S, Tirosh I, Wortman I, Rozenblatt-Rosen O, Wakimoto H, Rabkin SD, Cahill DP, Nahed BV, Curry WT, Martuza RL, Chi AS, Kasif $S$ and Shalek AK: Reconstructing and reprogramming the tumor-propagating potential of glioblastoma stem-like cells. Cell 157(3): 580-594, 2014.

20 Koh Y, Park I, Sun CH, Lee S, Yun H, Park CK, Park SH, Park JK and Lee SH: Detection of a distinctive genomic signature in rhabdoid glioblastoma, a rare disease entity identified by whole exome sequencing and whole transcriptome sequencing. Transl Oncol 8(4): 279-287, 2015.

21 Hashizume R, Gupta N, Berger MS, Banerjee A, Prados MD, Ayers-Ringler J, James CD and VandenBerg SR: Morphologic and molecular characterization of ATRT xenografts adapted for orthotopic therapeutic testing. Neuro Oncol 12(4): 366-376, 2010 .

Received April 6, 2018

Revised May 1, 2018

Accepted May 2, 2018 